\title{
Development of Secondary Processing Data Methods under Single Point Thunderstorm Activity Monitoring
}

\author{
Anatoly Panyukov ${ }^{\text {T }}$, Alexander Bogushov ${ }^{2}$ \\ ${ }^{1}$ School of Electronic Engineering and Computer Science, South Ural State University, 454080, Russian Federation \\ ${ }^{2}$ Institute of Natural Sciences and Mathematics, South Ural State University, 454080, Russian Federation
}

\section{A R T I C L E I N F O}

Article history:

Received: 31 August, 2020

Accepted: 25 November, 2020

Online: 14 December, 2020

\section{Keywords:}

Single-point lightning

Sensor pre-storm radiation

Equivalent electric dipole

Thunderstorm simulation

Passive monitoring

Visualization

\begin{abstract}
A B S T R A C T
Forecasting the occurrence and development of thunderstorms near insured mobile objects, such as aircraft carriers, oil tankers, requires the usage of appropriate single-point monitoring systems (SPMS) of thunderstorm activity. These systems must be small and included in the equipment of the objects. A SPMS based on EH location finder meets the requirements of small dimensions. But the primary processing algorithms for them used to determine the location of the radiation source lead to errors in the calculation of the bearing per atmospheric discharge. This inevitably occurs due to the presence of an anomalous component of the magnetic field of an arbitrarily oriented dipole. The error depends on the orientation of the equivalent dipole of the radiation source. If the dipole moment is vertical, the error is minimal, otherwise the direction finding error is completely determined by the horizontal projection of the equivalent dipole and can be equal to $\pi$ radians. Information of a thunderstorm activity collected by SPMS can be used by secondary processing algorithms to identify a real location of the thunderstorm foci. It is especially important when processing pre-storm radiation which consist of intraand inter-cloud discharges. Secondary processing algorithms reduce errors by analyzing the parameters of all registered lightning discharges over a certain time interval. The proposed algorithms divide the entire space into a set of three-dimensional cells and determine the their grade of membership to a thundercloud or its projection. The presented methods for analyzing the location parameters of radiation sources defined across the entire set of received signals and ways to display them reduce the uncertainty of determining the coordinates of the thunderstorm location. Processing of pre-thunderstorm radiation is essential for predicting the development of thunderstorms.
\end{abstract}

\section{Introduction}

Predicting the occurrence and development of a thunderstorm near the insured object requires the development of appropriate singlepoint systems for monitoring thunderstorm activity. For mobile insured objects, such as aircraft carriers, oil tankers, these systems must be small and included in the equipment of the objects [1]. Primary processing algorithms which used to determine location of the radiation source can lead to errors in calculation the bearing of discharge. It inevitably occurs in a single-point monitoring systems (SPMS). The error depends on the orientation of the equivalent dipole of the radiation source. If the dipole moment is vertical then the error is minimal, otherwise the direction finding error is completely determined by the horizontal projection of the equivalent dipole and can be equal to $\pi$ radians.
Information of a thunderstorm activity collected by a SPMS can be used by secondary processing algorithms to identify a real location of the thunderstorm foci. It is particularly critical when processing pre-storm radiation which consist of intra- and inter-cloud discharges. Secondary processing algorithms can reduce the uncertainty of determining the coordinates of the thunderstorm location by analyzing the location parameters of radiation sources that found for a time interval. This approach has been announced at "2019 Russian Open Conference on Radio Wave Propagation (RWP), Kazan, Russia" [2] in the field of study that deals with electromagnetic methods of lightning detection. This paper is an original extension of this work.

Each cloud and ground lightning flash consist of many individual physical processes. These processes can be associated with

*Corresponding Author: Anatoly Panyukov, Email: anatoly.panyukov@gmail.com 
characteristic electric and magnetic fields. Lightning emits significant electromagnetic energy in the radio-frequency range from below $1 \mathrm{~Hz}$ to near $300 \mathrm{MHz}$. This electromagnetic radiation can be detected by lightning locating systems. Lightning data have utility in different areas of importance meteorological service organizations. Typically, meteorological organizations use this data to protect life and property. Commercial organizations use lightning data to provide weather warnings, forecasts to aviation, agriculture, energy, mass media.

There are plenty of lightning locating systems that uses different radio-frequency ranges, for example, the very low-frequency (VLF) range from 3 to $30 \mathrm{kHz}$, the low-frequency (LF) range from 30 to $300 \mathrm{kHz}$. Accurate lightning locating systems necessarily employ multiple sensors. But single-point passive monitoring system are also popular as they are inexpensive, mobile and do not require communication. Many companies are engaged in the production of such devices [3]-[6].

A challenging problem which arises in this domain is processing pre-thunderstorm radiation (that is before the first lightning flash). During the project ISTC 1822 [7] were recorded 2.5 million atmospherics and less than $10 \%$ were defined as a lightning discharge, the rest radiation were classified as pre-thunderstorm radiation. Algorithms used in multiple sensors systems require registration of the atmospherics by several sensors, it is not always possible. Otherwise, single-point systems generally filtered out this data or it processed incorrectly.

Algorithms to determine location of the radiation source uses the magnetic direction finding (MDF) technique to calculate the bearing of discharge. It works really well for multistation systems especially with combination with time of arrival (TOA) technique. But using MDF technique in SPMS leads to significant errors for the discharges whose equivalent dipole has an orientation different from vertical.

In general, the results of the project showed possibility of creating a new generation of passive thunderstorm detection systems to extend their functionality. One way to overcome these problems is not only revision of the technical characteristics of sensors, but also development of new mathematical models and algorithms for analyzing thunderstorm activity. The problem of identifying the position of thunderstorm foci is practically important to forecasting the development of thunderstorm. The registration of the cloud radiation by a single-point lightning protection system, for the purposes of forecasting thunderstorm development, can be reached by expansion of receiving hardware dynamic range and development of mathematical and software.

The standard model for the lightning discharge is based on a vertical dipole that cannot adequately represent all discharges. Modern methods of analyzing the electromagnetic radiation source (EMRS) field allow to determine the parameters of the EMRS location by many algorithms based on the dipole as a model of the EMRS [1], [8]-[10]. A more correct approach is to simulate a lightning discharge as a arbitrarily oriented dipole and it possible by using special a group of $E-H$ methods that allow arbitrary orientation of dipoles [11, 12]. These algorithms allow us to estimate the position parameters of this dipole: the distance to the radiation source and the pseudo bearing, and can be used for processing prethunderstorm radiation. In this case, signals from various sources of electromagnetic radiation are registered, the appropriate mathematical model of which is an arbitrarily oriented electric dipole. Processing signals from dipoles with a predominant horizontal projection leads to uncertainty in the estimation of the bearing on the [2, 12] discharge, which significantly reduces the quality of dipoles location detection. One way to reduce this uncertainty is to analyze the information received from all dipole sources.

The aim of the work is describing approach for the secondary processing of monitoring results of thunderstorm activity in VLF range, allowing to get plausible estimates of the location of a thunderstorm center based on its pre-storm radiation.

A review of the status of passive storm monitoring systems and the demonstration of the use of lightning-position detection systems for the passive radar of hazardous meteorological phenomena are presented in [8]. Modern methods for analyzing the field, allowing to determine the parameters of the EMRS, characterizing its location and orientation are presented in the works [12].

\section{Primary processing of EMRS signal}

Figure 1 shows the geometry of the problem: the earth's surface is plane $O x y$, the observation point is origin $O$, the polar coordinates of the radiation source are $r, \psi, \theta$, and the angular coordinates of the radiation source dipole moment are $\psi_{0}, \theta_{0}$.



Figure 1: Geometry of the problem

There are a dipole $\mathbf{P}_{\mathbf{0}}$ and an imaginary dipole $\mathbf{P}_{\mathbf{1}}$ which is the mirror image of the dipole $\mathbf{P}_{\mathbf{0}}$. $\mathbf{P}_{\mathbf{0}}$ results a field $(\mathbf{E}, \mathbf{H})$ consisting of the field $\left(\mathbf{E}\left(\mathbf{P}_{\mathbf{0}}\right), \mathbf{H}\left(\mathbf{P}_{\mathbf{0}}\right)\right)$ of the source $\mathbf{P}_{\mathbf{0}}$, and the reflected field represented by the field $\left(\mathbf{E}\left(\mathbf{P}_{\mathbf{1}}\right), \mathbf{H}\left(\mathbf{P}_{\mathbf{1}}\right)\right)$ of the $\mathbf{P}_{\mathbf{1}}$.

The electric $\mathbf{E}$ and magnetic $\mathbf{H}$ components of the field in terms of the Cartesian system of coordinates with origin at the observation 
point $O$ and the $O_{z}$ axis being the normal of the bounding plane have representation [9]

$$
E_{x}=E_{y}=H_{z}=0,
$$

$$
\begin{gathered}
E_{z}=\frac{1}{2 \pi \epsilon_{0} c^{2} r}\left\{p^{\prime \prime}\left[\cos \theta \sin \theta \sin \theta_{0} \cos \left(\psi-\psi_{0}\right)-\sin ^{2} \theta \cos \theta_{0}\right]+\right. \\
\left.+\left(p^{\prime} \alpha+p \alpha^{2}\right)\left[3 \sin \theta \cos \theta \sin \theta_{0} \cos \left(\psi-\psi_{0}\right)\right]\right\}= \\
=\frac{w}{2 \pi \epsilon_{0} c^{2} r}\left[p^{\prime \prime} u+\left(p^{\prime} \alpha+p \alpha^{2}\right)(3 u-2 v)\right], \quad
\end{gathered}
$$

$$
\begin{array}{r}
H_{x}=\frac{1}{2 \pi c^{2} r}\left(\sin \theta \cos \theta_{0} \sin \psi-\cos \theta \sin \theta_{0} \sin \psi_{0}\right)\left(p^{\prime \prime}+p^{\prime} \alpha\right)= \\
=\frac{w \sin \varphi}{2 \pi c^{2} r}\left(p^{\prime \prime}+p^{\prime} \alpha\right),
\end{array}
$$

$H_{y}=\frac{1}{2 \pi c^{2} r}\left(-\sin \theta \cos \theta_{0} \cos \psi+\cos \theta \sin \theta_{0} \cos \psi_{0}\right)\left(p^{\prime} \alpha+p^{\prime \prime}\right)=$

$$
\begin{array}{r}
=-\frac{w \cos \varphi}{2 \pi c^{2} r}\left(p^{\prime} \alpha+p^{\prime \prime}\right), \\
\alpha=\frac{c}{r}, \quad u=\sin \theta \cos (\phi-\psi), \quad v=\frac{\sin \left(\phi-\psi_{0}\right)}{\sin \left(\psi-\psi_{0}\right)}, \\
\tan \varphi=\frac{\sin \theta \cos \theta_{0} \sin \psi-\cos \theta \sin \theta_{0} \sin \psi_{0}}{\sin \theta \cos \theta_{0} \cos \psi-\cos \theta \sin \theta_{0} \cos \psi_{0}},
\end{array}
$$

$c$ is speed of light in vacuum, $\epsilon_{0}$ is permittivity of vacuum.

The values of the parameters $r, \varphi, u, v$ are measured as a result of primary processing, for each pulse signal received from a radiation source [9]. The quality of estimating the location of an individual source is quite low. Really, a vertical dipole (that is when $\left.\theta_{0}=0\right)$ have

$$
\varphi=\psi, \quad \sin \theta=u=\frac{1}{v} .
$$

In other cases (that is for an inclined dipole) there is an uncertainty in determining the coordinates $\psi$ and $\theta$. As shown in the article in [9] they are bounded by

$$
\begin{gathered}
\arcsin u \leq \theta \leq \frac{\pi}{2}, \\
\varphi-\arccos u \leq \psi \leq \varphi+\arccos u .
\end{gathered}
$$

The probability density for angles $\psi$ and $\theta$ are

$$
\begin{aligned}
& f_{\psi}(\eta)=\frac{s}{2 \widehat{\psi}\left[s^{2} \cos ^{2}(\varphi-\eta)+\sin ^{2}(\varphi-\eta)\right]}, \\
& -\arccos u \leq \eta-\varphi \leq \arccos u, \\
& f_{\theta}(\zeta)=\frac{s u \cos \zeta \sin \zeta}{\widehat{\psi}\left(s^{2} u^{2}-u^{2}+\sin ^{2} \zeta\right) \cdot \sqrt{\sin ^{2} \zeta-u^{2}}}, \\
& \arcsin u \leq \zeta \leq \frac{\pi}{2} .
\end{aligned}
$$

where

$$
s=\left|\frac{1-v u}{u v}\right|, \quad \widehat{\psi}=\arctan \left(\frac{1}{s} \tan \arccos u\right) .
$$

It's easy to see that for $s<1$, that is for $\theta_{0}$ approaching the vertical,

$$
\begin{gathered}
\varphi=\arg \max _{\eta:-\arccos u \leq \eta-\varphi \leq \arccos u} f_{\psi}(\eta), \\
\arcsin u=\arg \max _{\zeta: \arcsin u \leq \zeta \leq \frac{\pi}{2}} f_{\theta}(\zeta),
\end{gathered}
$$

in other words, the maximum likelihood estimates are the same as for the vertical dipole: $\psi=\varphi$ and $\theta=\arcsin u$; and for $s \geq 1$, that is for orientation with a significant horizontal component, poor angular resolution is gained.

An adequate mathematical model of the pre-thunderstorm EMRS is an inclined electric dipole with a predominance of the horizontal component. Processing signals from dipoles with a predominant horizontal projection leads to uncertainty in the estimation of the angle coordinates of the [2, 9] discharge, which significantly reduces the quality of dipoles location detection.

The multipoint system of passive monitoring of thunderstorm activity not only removes the uncertainty of location [9], but also allows to determine the orientation of the dipole [13, 14]. Nevertheless, the improvement of forecasting methods using single-point systems remains relevant [15, 16].

One way to reduce the uncertainty of thunderstorm location is to analyze the information received from all dipole sources for time $T$.

The purpose of secondary processing is to estimate the location of the thunderstorm center by information about all dipoles location parameters for time $T$.

\section{Secondary processing of EMRS signals from thunderstorm foci}

It is known that the Cartesian coordinates $x, y$ of the possible position of the EMRS satisfy the condition [9]:

$$
x \cos \varphi+y \sin \varphi=r \sin \theta \cos (\varphi-\psi)=r u,
$$

which determines the $O X Y$ projection of the EMRS possible positions set. The coordinates of the possible location EMRS belong to the set of solutions of the system of equations

$$
x \cos \varphi+y \sin \varphi=r u, \quad x^{2}+y^{2}+z^{2}=r^{2} .
$$

Let $S$ be set of all solutions of 15 system. In general, $S$ is multiple. Entire space divided into $4 N^{3}$ cells:

$$
\begin{gathered}
C(i, j, k)=\left\{\begin{array}{l|l}
(x, y, z) & \begin{array}{l}
i \Delta \leq x \leq(i+1) \Delta \\
j \Delta \leq y \leq(j+1) \Delta \\
k \Delta \leq z \leq(k+1) \Delta
\end{array}
\end{array}\right\}, \quad k=0,1, \ldots, N, \\
i, j=-N,-N+1, \ldots,-1,0,1, \ldots N .
\end{gathered}
$$

The OXY plane is divided into flat cells

$$
\begin{aligned}
C(i, j)=\{(x, y): & i \Delta \leq x \leq(i+1) \Delta, \Delta \leq y \leq(j+1) \Delta, \\
& i, j=-N,-N+1, \ldots,-1,0,1, \ldots N\},
\end{aligned}
$$

and $\mathrm{OZ}$ axis of the coordinate system $O X Y Z$ to be divided into segments

$$
C(k)=\{z: k \Delta \leq z \leq(k+1) \Delta\}, \quad k=0,1, \ldots, M .
$$


In the future we will use the following notation:

$$
C_{O X Y Z}(r, u, \varphi)=\{(i, j, k) \mid C(i, j, k) \cap S \neq \emptyset\}
$$

is set of cells in which the EMRS can be locate with a non zero probability;

$$
C_{O X Y}(r, u, \varphi)=\{(i, j) \mid \exists k: C[i, j, k] \cap S \neq \emptyset\}
$$

is $O X Y$ projection of set $C_{O X Y Z}(r, u, \varphi)$;

$$
C_{O Z}(r, u, \varphi)=\{k \mid \exists C(i, j): C(i, j, k) \cap S \neq \emptyset\}
$$

is $O Z$ projection of set $C_{O X Y Z}(r, u, \varphi)$.

For each cell containing solutions of the 15 system, we find the probability that the EMRS belongs to this cell when some hypothesis is fulfilled. In the future, we will use the found probabilities to build a three-dimensional map of the membership level of cells that are part of the thunderstorm center.

\subsection{Hypothesis $H_{1}$ : \\ Uniform probability distribution on $C_{O X Y}(r, u, \varphi)$}

It is following from (15) that on the $O X Y$ plane, the valid points farthest from the origin of the coordinates are $\left(x_{1}, y_{1}\right)$ and $\left(x_{2}, y_{2}\right)$

$$
\begin{gathered}
x_{1,2}=r\left(u \cos \varphi \mp \sqrt{1-u^{2}} \sin \varphi\right), \\
y_{1,2}=r\left(u \sin \varphi \pm \sqrt{1-u^{2}} \cos \varphi, \quad z_{1,2}=0 .\right.
\end{gathered}
$$

The coordinates $x, y$ for all possible ERMS positions belong to segment $\left[\left(x_{1}, y_{1}\right),\left(x_{2}, y_{2}\right)\right] \subset O X Y$ between the points $\left(x_{1}, y_{1}\right)$ and $\left(x_{2}, y_{2}\right)$. The all possible radiation source locations can be represented in a parametric form as the set of points:

$$
\mathcal{L}(r, u, \varphi)=\left\{\begin{array}{c}
x(\lambda)=r u \cos \varphi+ \\
+r(2 \lambda-1) \sqrt{1-u^{2}} \sin \varphi, \\
y(\lambda)=r u \sin \varphi+ \\
+r(1-2 \lambda) \sqrt{1-u^{2}} \cos \varphi, \\
z(\lambda)=2 r \times \\
\times \sqrt{\lambda(1-\lambda)\left(1-u^{2}\right)}
\end{array} \mid 0 \leq \lambda \leq 1\right\} .
$$

Let

$$
\Lambda=\left\{0, \frac{1}{N}, \frac{2}{N}, \ldots, \frac{N-1}{N}, 1\right\} .
$$

It is following from t20 that points $(x(\lambda), y(\lambda)), \lambda \in \Lambda$ are evenly distributed on the segment $\left[\left(x_{1}, y_{1}\right),\left(x_{2}, y_{2}\right)\right]$, and

$$
\left[\left(x_{1}, y_{1}\right),\left(x_{2}, y_{2}\right)\right] \subset \bigcup_{(i, j) \in C_{O X Y}(r, u, \varphi)} C(i, j) .
$$

If the hypothesis on uniform probability distribution on $C_{O X Y}(r, u, \varphi)$ is accepted, there are equalities

$$
\begin{aligned}
& C_{\text {OXYZ }}(r, u, \varphi)= \\
& \quad=\left\{(i, j, k): i=\left\lfloor\frac{x(\lambda)}{\Delta}\right\rfloor, j=\left\lfloor\frac{y(\lambda)}{\Delta},\right\rfloor k=\left\lfloor\frac{z(\lambda)}{\Delta}\right\rfloor, \lambda \in \Lambda\right\},
\end{aligned}
$$

$$
C_{O X Y}(r, u, \varphi)=\left\{(i, j): i=\left\lfloor\frac{x(\lambda)}{\Delta}\right\rfloor, j=\left\lfloor\frac{y(\lambda)}{\Delta}\right\rfloor, \lambda \in \Lambda\right\} .
$$

Let

$$
\begin{gathered}
N(i, j, k)=\operatorname{card}\{\lambda \in \Lambda:(x(\lambda), y(\lambda), z(\lambda) \in C(i, j, k))\}, \\
N(i, j)=\sum_{k \in C_{O Z}(r, u, \varphi)} N(i, j, k), .
\end{gathered}
$$

Then, the probability of EMRS in cell $C[i, j, k]$ is equal to

$$
\mathrm{P}_{O X Y}(i, j, k)=\frac{N(i, j, k)}{N(i, j) \cdot N} .
$$

\subsection{Hypothesis $\mathrm{H}_{2}$ : \\ Uniform probability distribution on $C_{O Z}(r, u, \varphi)$}

It follows from (15) that the set of points of all possible locations of EMRS can be represented in a parametric form other than 20 :

$$
\mathcal{L}(r, u, \varphi)=\left\{\begin{array}{c}
x(z)=r u \cos \varphi \pm \sqrt{r^{2}\left(1-u^{2}\right)-z^{2}} \sin \varphi, \\
y(z)=r u \sin \varphi \mp \sqrt{r^{2}\left(1-u^{2}\right)-z^{2}} \cos \varphi, \\
z: 0 \leq z \leq \sqrt{r^{2}\left(1-u^{2}\right)}
\end{array}\right\}
$$

Let

$$
M=\left\lfloor\frac{\sqrt{r^{2}\left(1-u^{2}\right)}}{\Delta}\right\rfloor, \quad Z=\{0, \Delta, 2 \Delta, \ldots, M \cdot \Delta\} .
$$

It is following from (22) that points $z \in Z$ are evenly distributed on the segment

$$
\left[0, \sqrt{r^{2}\left(1-u^{2}\right)}\right] \subset \bigcup_{k: \in C_{O Z}(r, u, \varphi)} C[k] .
$$

If the hypothesis on uniform probability distribution on $C_{O Z}(r, u, \varphi)$ is accepted, there are equalities

$$
\begin{aligned}
& C_{\text {OXYZ }}(r, u, \varphi)= \\
& =\left\{(i, j, k): i=\left\lfloor\frac{x(z)}{\Delta}\right\rfloor, j=\left\lfloor\frac{y(z)}{\Delta},\right\rfloor k=\left\lfloor\frac{z}{\Delta}\right\rfloor, z \in Z\right\}, \\
& C_{O X Y}(r, u, \varphi)=\left\{(i, j): i=\left\lfloor\frac{x(z)}{\Delta}\right\rfloor, j=\left\lfloor\frac{y(z)}{\Delta}\right\rfloor, z \in Z\right\} .
\end{aligned}
$$

Let

$$
\begin{gathered}
M(i, j, k)=\operatorname{card}\{z \in Z:(x(z), y(z), z) \in C(i, j, k)\} \\
M(k)=\sum_{[i, j] \in C_{O X Y}(r, u, \varphi)} M(i, j, k), \quad k: k \cdot \Delta \in Z
\end{gathered}
$$

then

$$
\mathrm{P}_{O Z}(i, j, k)=\frac{M(i, j, k)}{M(k) \cdot M} .
$$




\subsection{Hypothesis $\mathrm{H}_{3}$ : \\ Uniform probability distribution on $C_{O X Y Z}(r, u, \varphi)$}

Since 20p and 21) give different representations of set $\mathcal{L}(r, u, \varphi)$, there are also two ways to represent a set of cells with a non-zero probability of having the EMRS:

$$
\begin{aligned}
C_{O X Y Z}(r, u, \varphi)=\left\{(i, j, k): \mathrm{P}_{O X Y}(i, j, k)>0\right\}= \\
=\left\{(i, j, k): \mathrm{P}_{O Z}(i, j, k)>0\right\} .
\end{aligned}
$$

If the hypothesis on uniform probability distribution on $C_{O X Y Z}(r, u, \varphi)$ is accepted, we have

$$
\mathrm{P}_{O X Y Z}(i, j, k)=\frac{1}{\operatorname{card} C_{O X Y Z}(r, u, \varphi)}, \quad(i, j, k) \in C_{O X Y Z}(r, u, \varphi) .
$$

\subsection{Three-dimensional map of the level belonging of thunderstorm foci to space cells}

So, for each cell containing solutions of the 15 system, we found the probability that the EMRS belongs to this cell when hypothesis $H \in\left\{H_{1}, H_{2}, H_{3}\right\}$ is fulfilled. Let us use the found probabilities to build a three-dimensional map of the membership level of cells that are part of the thunderstorm center when hypothesis $H$ is fulfilled.

The set of discharges registered for the time period $T$ is defined as $R(T)$. The cell grade membership to thunderstorm of the radiation source defined as the sum of all of radiation during this period.

$$
\operatorname{grade}_{H}^{R(T)}(i, j, k)=\frac{\sum_{d \in R(T)} \mathrm{P}_{H}(i, j, k)(d)}{\max _{(l, m, n)} \sum_{d \in R(T)} \mathrm{P}_{H}(l, m, n)(d)} .
$$

Further we demonstrate the possibility to estimate the thunderstorm center location by (23) formula applying simulation.

\section{Simulation}

Let's define two artificial thunderstorms $R_{1}(T)$ and $R_{2}(T)$ for modeling purposes. All discharges of the thunderstorms will have the same location $R=60 \mathrm{~km}, \Delta=10 \mathrm{~km}, \psi=\pi / 4, \theta=\pi / 3$.

Figures 38 demonstrate the observation point $(x=0, y=$ $0, z=0)$ and the thunderstorm location cell is $C(i=3, j=3, k=3)$ (marked with a blue square). Cell color shows the cell grade membership level to the thunderstorm (see figure 2).



Figure 2: Scale of the level of belonging of a cell to a thunderstorm center

The $R_{1}(T)$ emits 256 pulse signals from dipole radiation sources with orientation $\psi_{0} \in\{\pi k / 16: k=1,2, \ldots, 32\}, \theta_{0} \in\{\pi k / 8$ : $k=0,1, \ldots, 8\}$, that is EMRS are different incline dipoles, which is typical for a thunderstorm center. Location parameters values $r, u, v, \varphi$ are calculated by (5)-(6) formulas.

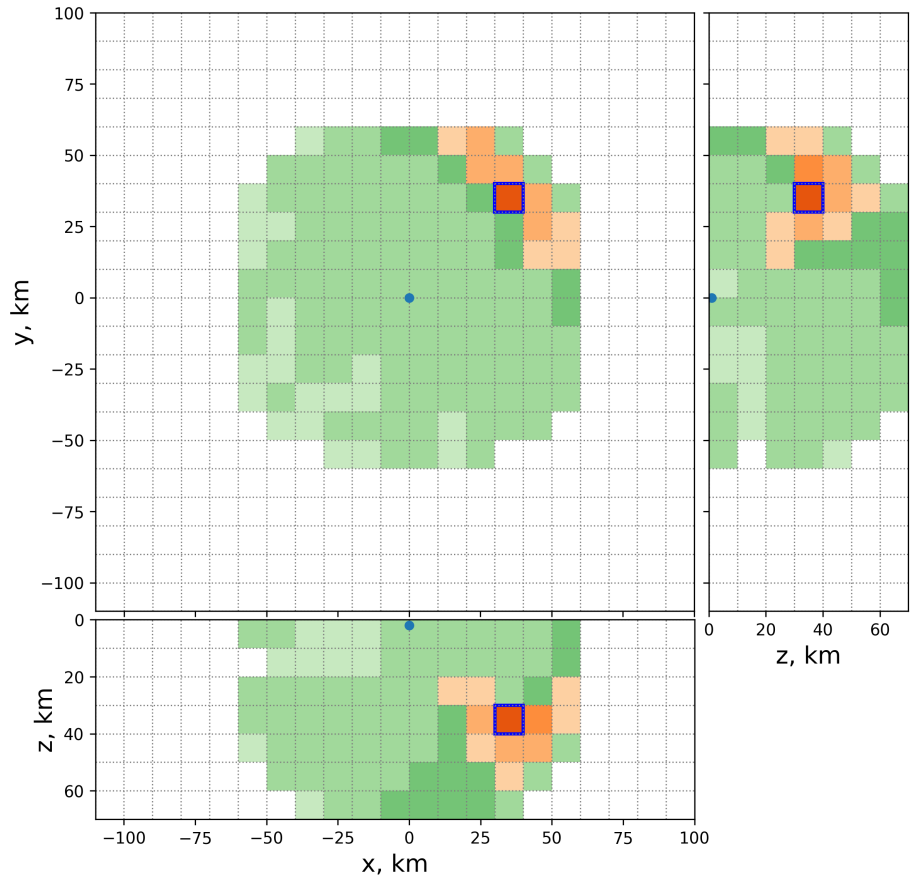

Figure 3: Map of $R_{1}(T)$ under hypothesis $H_{1}$

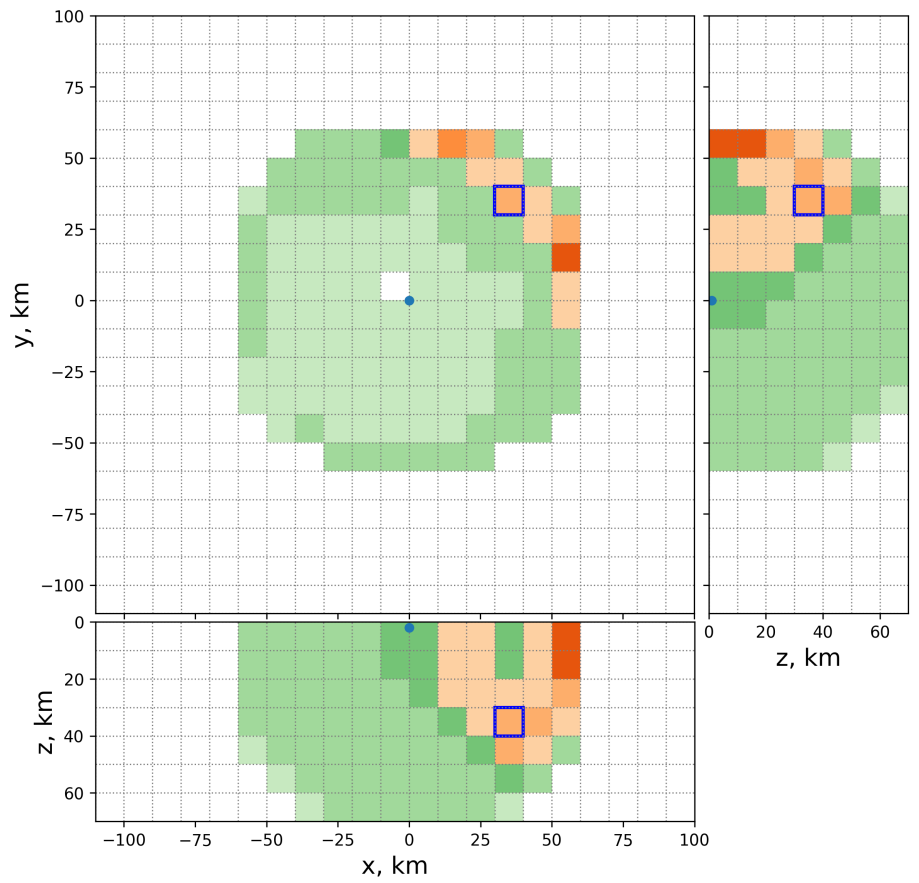

Figure 4: Map of $R_{1}(T)$ under hypothesis $H_{2}$

Figures 3,8 demonstrate three projections of the threedimensional map of thunderstorm foci $R_{l}(T), l \in\{1,2\}$ level belonging to space cells under hypothesis $H \in\left\{H_{1}, H_{2}, H_{3}\right\}$

$$
\begin{aligned}
& \operatorname{grade}_{H}^{R}(T)(i, j)=\sum_{k} \operatorname{grade}_{H}^{R}(T)(i, j, k), \\
& \operatorname{grade}_{H}^{R}(T)(i, k)=\sum_{j} \operatorname{grade}_{H}^{R}(T)(i, j, k),
\end{aligned}
$$




$$
\operatorname{grade}_{H}^{R}(T)(j, k)=\sum_{i} \operatorname{grade}_{H}^{R}(T)(i, j, k) .
$$

Figure 3 demonstrates the belonging of desired cell to cell with maximum level of membership. Figure 4 demonstrates that the desired cell does not belong to cells with maximum level of membership. Figure 5 demonstrates the belonging of desired cell to cell with maximum level of membership.

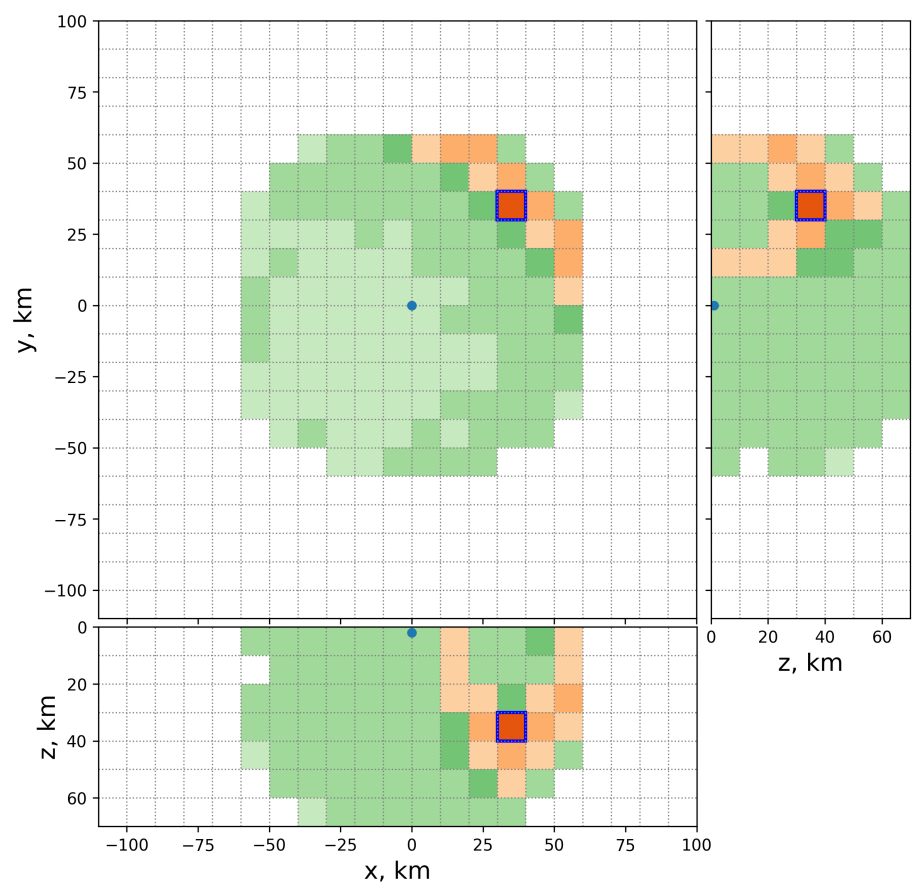

Figure 5: Map of $R_{1}(T)$ under hypothesis $H_{3}$

Figures 3, 5 make it possible to identify the desired cell $C(i=3, j=3, k=3)$. Figure 4 does not allow to identify the desired cell at all.

Table 1 shows cells with grade membership level to the thunderstorm $R_{1}(T)$ no less 0.9 and similar to visual analysis of the projections hypothesis $H_{1}$ and $H_{3}$ found the desired cell.

Table 1: Cells with grade membership level to the thunderstorm $R_{1}(T)$ no less 0.9

\begin{tabular}{|c|c|}
\hline Hypothesis & $C_{\text {OXYZ }}(r, u, \varphi) \cap\left\{(i, j, k): \operatorname{grade}_{H}^{R_{1}(T)}(i, j, k)>0.9\right\}$ \\
\hline$H_{1}$ & $\{(3,3,3),(3,2,3),(4,3,3)\}$ \\
$H_{2}$ & $\{(1,5,0),(1,5,1),(3,3,4),(5,1,0),(5,1,1)\}$ \\
$H_{3}$ & $\{(3,3,3)\}$ \\
\hline
\end{tabular}

But the best hypothesis is hypothesis $H_{3}$, because it will allow to identify the cell with a thunderstorm center uniquely and accurately.

The $R_{2}(T)$ has the same location as $R_{1}(T)$ but emits only 100 pulse signals from dipole radiation sources with orientation $\psi_{0} \in\{2 \pi k / 100: k=1,2, \ldots, 100\}, \theta_{0}=\pi / 2$, that is EMRS are different horizontal dipoles, which is typical for a pre-storm center. Location parameters values $r, u, v, \varphi$ are calculated by (5)-(6) formulas.

Figures 68 demonstrate three projections of the threedimensional map of thunderstorm foci $R_{2}(T)$ level belonging to space cells under hypothesis $H \in\left\{H_{1}, H_{2}, H_{3}\right\}$.
Figure 6 demonstrates that the desired cell does not belong to cells with maximum level of membership.



Figure 6: Map of $R_{2}(T)$ under hypothesis $H_{1}$

Figure 7 demonstrates that the desired cell does not belong to cells with maximum level of membership.

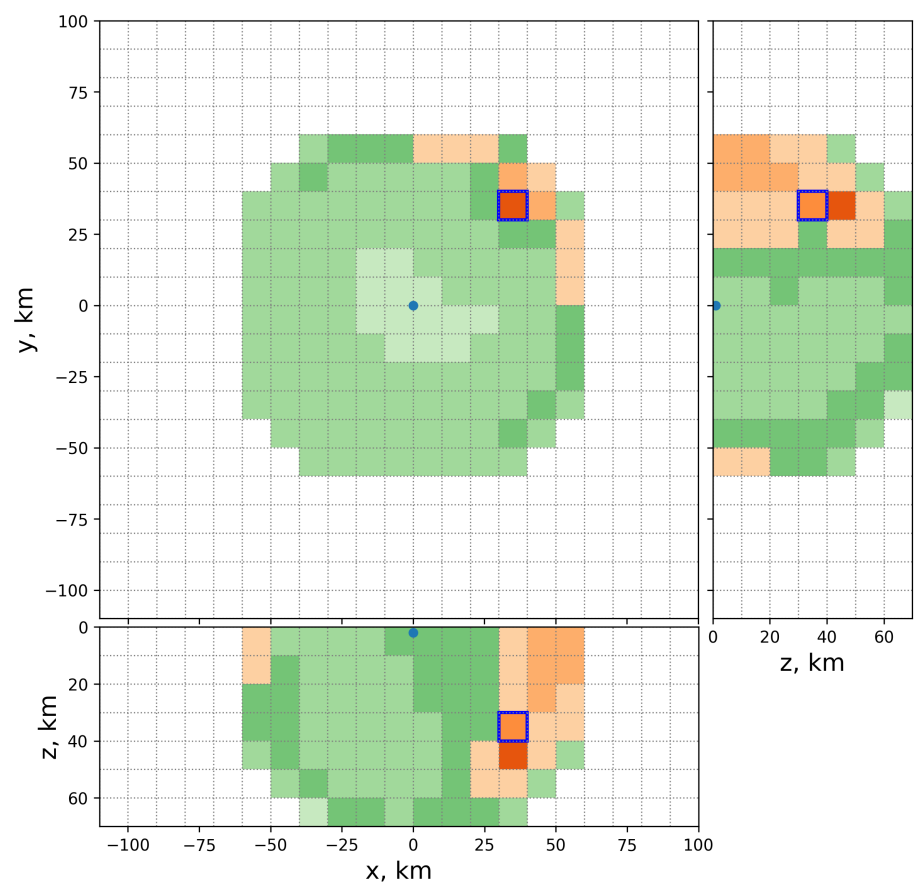

Figure 7: Map of $R_{2}(T)$ under hypothesis $H_{2}$

Figure 8 demonstrates that the desired cell belong to cells with maximum level of membership only. 


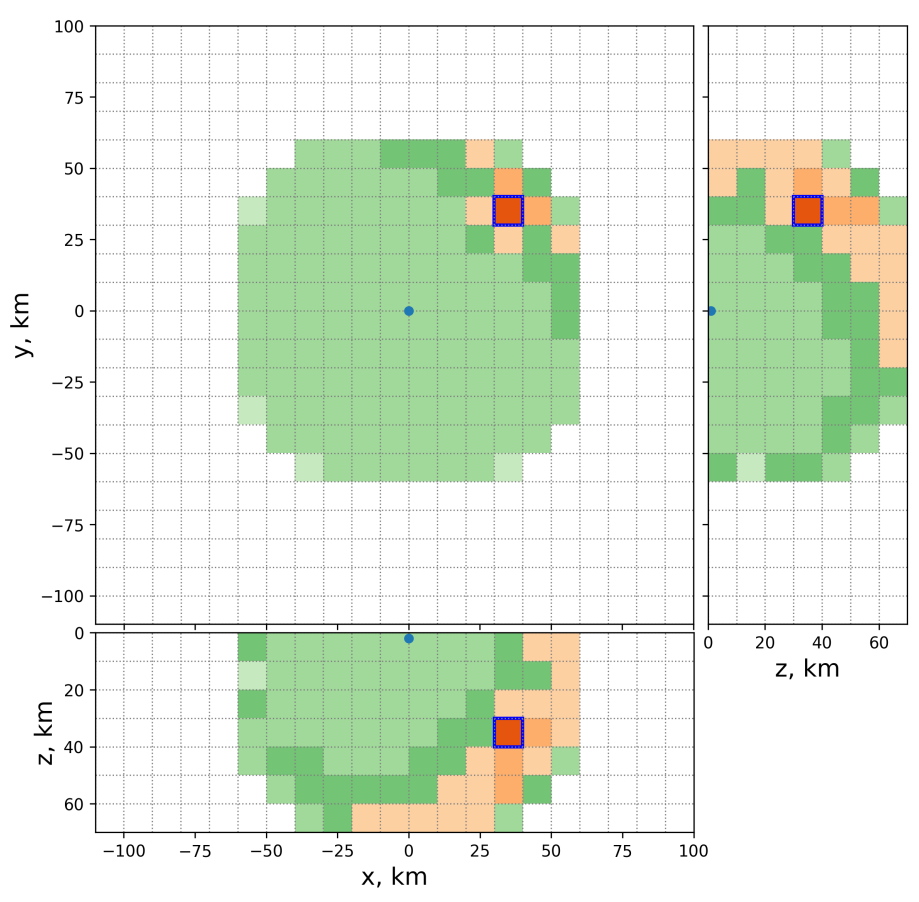

Figure 8: Map of $R_{2}(T)$ under hypothesis $H_{3}$

Table 2 also shows the best hypothesis is $H_{3}$. Hypotheses $H_{1}$ and $H_{2}$ maps do not contains the desired cell and does not allow to unambiguously establish the location of the foci.

Table 2: Cells with grade membership level no less 0.9 to the thunderstorm $R_{2}(T)$

\begin{tabular}{|c|c|}
\hline Hypothesis & $C_{O X Y Z}(r, u, \varphi) \cap\left\{(i, j, k): \operatorname{grade}_{H}^{R_{2}(T)}(i, j, k)>0.9\right.$ \\
\hline$H_{1}$ & $\{(3,3,4)\}$ \\
$H_{2}$ & $\{(3,3,4)\}$ \\
$H_{3}$ & $\{(3,3,3),(3,3,4)\}$ \\
\hline
\end{tabular}

\section{Conclusion}

These findings provide a potential new technique to processing of the results of thunderstorm activity monitoring in the VLF range. The processing of all data from the SPMS during thunderstorm activity introduces a lot of uncertainty and makes it difficult to determine the real location of the thunderstorm center. The methods of analysis and visualization of the entire set of received signals presented in this paper reduce the uncertainty in determining the coordinates of the thunderstorm location. Processing of pre-thunderstorm radiation is significantly important to forecasting the development of thunderstorm.

The artificial single-point thunderstorm model described in the paper is a very simple, which is very convenient for research, however, a real thunderstorm can have a more complex structure, there- fore it is important to carry out modelling with more complex models of thunderstorms, for example, check the proposed approaches for their ability to identification of multiple foci. This is an interesting topic for future work.

In future work, investigating another hypothesis of probability that the EMRS belongs to cell might be important.

\section{References}

[1] A. V. Panyukov, A. K. Bogushov, "Single-point system of positioning storms in close zone," RU Patent 2,599,775, 2016.

[2] A. V. Panyukov, A. K. Bogushov, "A Method For Processing Data of Thunderstorm Activity Monitoring," in 2019 Russian Open Conference on Radio Wave Propagation (RWP), 556-559, 2019, doi:10.1109/RWP.2019.8810345.

[3] “Thunderstorm Electric Field Mill EFM550," 2020.

[4] “Thunderstorm Advanced Total Lightning Sensor LS7002," 2020.

[5] “Boltek LD-350 Lightning Detector," 2020.

[6] “Biral BTD-300 Thunderstorm Detector," 2020.

[7] "The International Science and Technology Center. Project; 1822. Development and Investigation of the Single-Point System for Lightning Location in the Range of SuperLong Waves," 2000.

[8] I. Kononov, I. Yusupov, N. Kandaratskov, "Analysis of One-Point Methods for Lightning-Discharge Passive Location," Radiophysics and Quantum Electronics, 56, 788-800, 2014, doi:10.1007/s11141-014-9481-1.

[9] A. V. Panyukov, "Estimation of the Location of an Arbitrary Oriented Dipole under Single-point Direction Finding," Journal of Geophysical Research, 101, 14997-14982, 1996, doi:10.1029/96JD00067.

[10] B. Taibin, "An Approach to Define Parameters for Localization of Thunderstorm," IEEE Antennas and Propagation Magazine, 48, 48-54, 2006, doi: 10.1109/MAP.2006.1650816.

[11] A. V. Panyukov, A. K. Bogushov, "The Spectral Statistical Method for Determining the Location Parameters of a Dipole Source of Electromagnetic Radiation," Radiophysics and Quantum Electronics, 59, 278-288, 2016, doi: 10.1007/s11141-016-9696-4.

[12] A. V. Panyukov, "Inverse Problem for an Electrical Dipole and the Lightning Location Passive Monitoring System," in M. S. Kandelousi, editor, Electric Field, chapter 13, IntechOpen, Rijeka, 2018, doi:10.5772/intechopen.74264.

[13] S. He, M. Popov, V. Romanov, "Explicit full identification of a transient dipole source in the atmosphere from measurement of the electromagnetic fields at several points at ground level," Radio Science, 35(1), 107-117, 2000, doi: https://doi.org/10.1029/1999RS002198.

[14] M. Popov, S. He, "Identification of a transient electric dipole over a conducting half space using a simulated annealing algorithm," Journal of Geophysical Research, 105, 20821-20831, 2000, doi:10.1029/2000JD900261.

[15] A. Leal, V. Rakov, E. Alves, M. Lopes, "Estimation of -CG lightning distances using single-station E-field measurements and machine learning techniques," 1-8, 2019, doi:10.1109/SIPDA47030.2019.9004484.

[16] "Vaisala. ALARM Automated Lightning Alert and Risk Management System," 2017. 